\title{
Identifying Ad-hoc Synchronization for Enhanced Race Detection
}

IPDPS - 20 April, 2010 Ali Jannesari / Walter F. Tichy

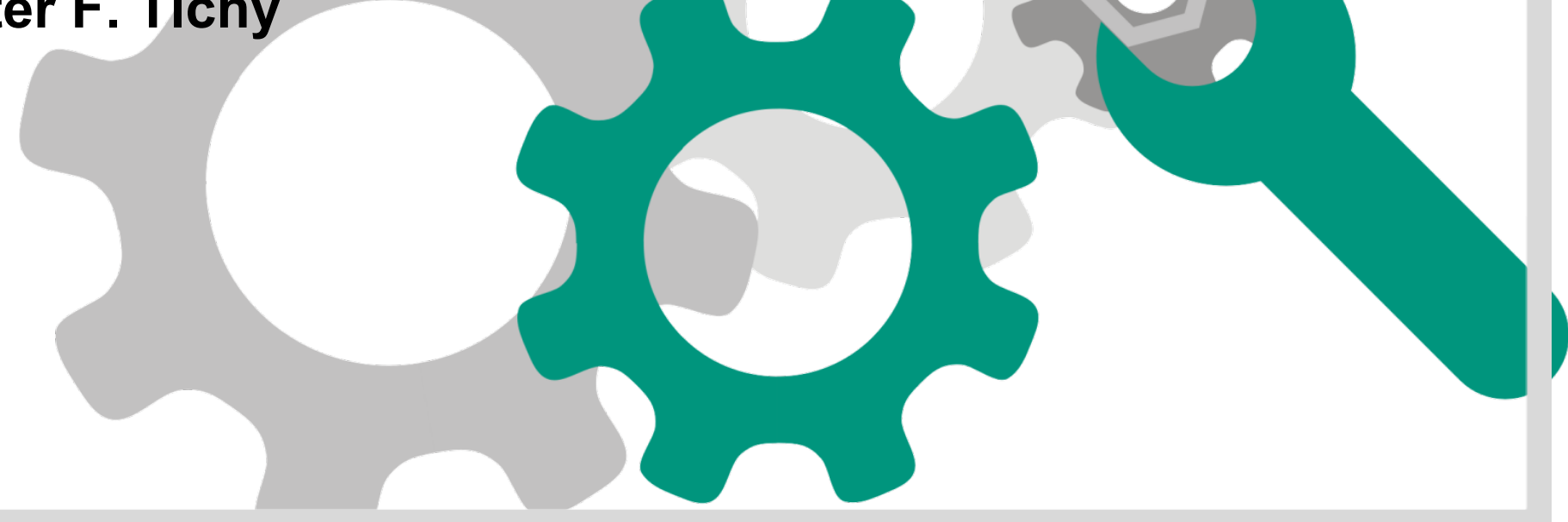




\section{Motivation}

- Data races (unsynchronized accesses to share variables) are a common defect in parallel programs.

- They are difficult to find.

- Race detectors are impractical

- They produce thousands to millions of false warnings.

- Programmers are overwhelmed by false positives.

- Why false positives?

- Ad-hoc, programmer-defined synchronizations

- Unknown synchronization libraries

- Detectors cannot reason about these, causing many false positives

- Contribution: how to handle user-defined synchronization and unknown synchronization libraries, reducing false positives. 


\section{What is a Data Race?}

- Two or more concurrent accesses to a shared location, at least one of them a write.

$$
\begin{aligned}
& \underline{\text { Thread } 1 \quad \underline{\text { Thread } 2}} \\
& X=0 \\
& \mathrm{X}++\quad \mathrm{T}=\mathrm{X}
\end{aligned}
$$




\section{Example - Data Race}

- First Interleaving: $\underline{\text { Thread } 1}$ Thread 2

$$
\text { 1. } \mathrm{X}=0
$$

2.

$$
\mathrm{T}=\mathrm{X}
$$

3. $\mathrm{X}++$

- Second Interleaving: Thread 1 Thread 2

$$
\begin{array}{ll}
\text { 1. } & \mathrm{X}=0 \\
\text { 2. } & \mathrm{X}++
\end{array}
$$

3.

$$
T=X
$$

- $\mathrm{T}==0$ or $\mathrm{T}==1$ ? 


\section{How Can Data Races be Prevented?}

- Explicit synchronization between threads:

- Locks

- Critical Sections

- Barriers

- Mutexes

- Semaphores

- Monitors

- Events (signal/wait)

- Etc.

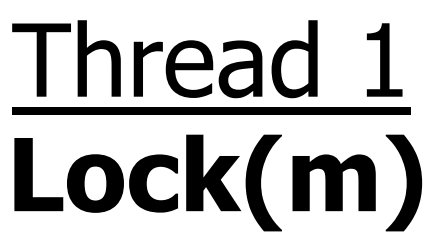

$X=0$

$\mathrm{X}++$

Unlock(m)
Thread 2

$\operatorname{Lock}(\mathbf{m})$

$\mathrm{T}=\mathrm{X}$

Unlock(m) 


\section{Detection Approaches}

- Static: perform a compile-time analysis of the code, reporting potential races.

- Dynamic: use tracing mechanism to detect whether a particular execution of a program actually exhibits dataraces

- The program must be instrumented with additional instructions to monitor shared variables and synchronization operations.

- Every shared variable has a shadow cell in which the race detector stores additional information. 


\section{Dynamic Data Race Detection}

- Dynamic Data Race Detection

- Lockset analysis

- Happens-before analysis

- Hybrids (combining Lockset and Happens-before) 


\section{Lockset Analysis}

- Observe all instances where a shared variable is accessed by a thread.

- Check whether the shared variable is always protected by the same lock.

- If variable isn't protected, issue a warning.

- The lockset for a variable is initially set to all locks occurring in program.

- Whenever a variable is accessed, remove all locks from the variable's lockset that are not currently protecting the variable.

- When the lockset is empty, issue a warning. 


\section{Lockset Analysis}

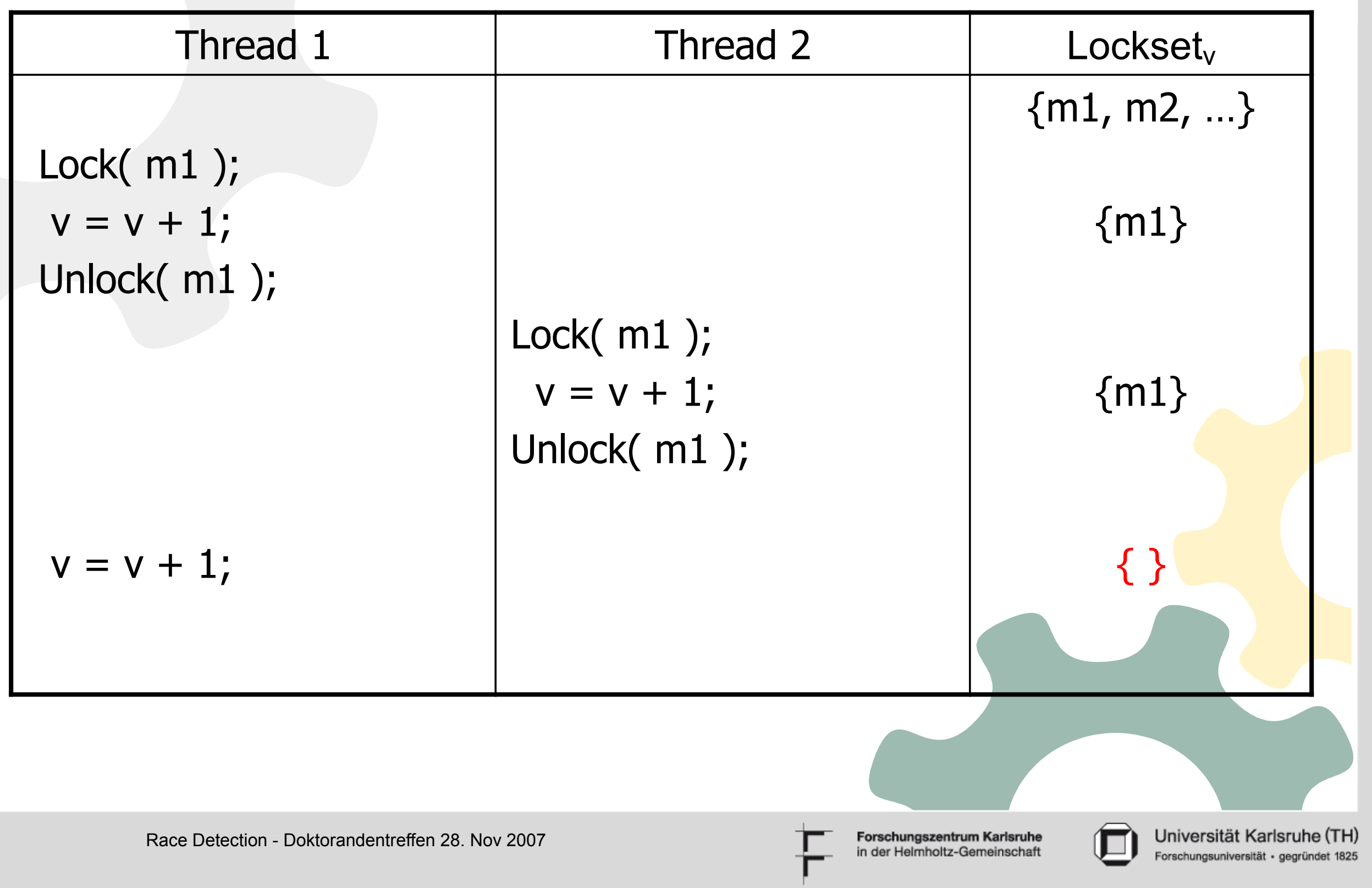




\section{Lockset - False Positives}

- The lockset algorithm will produce a false alarm in the following simple case:

- Not able to detect signal-wait operation

$$
\begin{aligned}
& \text { Thread } 1 \\
& \hline X=0 \\
& X++ \\
& \text { Signal(CV) }
\end{aligned}
$$$$
\text { Thread } 2
$$

\section{Wait(CV)}

$$
\mathrm{T}=\mathrm{X}
$$




\section{Happens-Before Relation}

- Based on Lamport's Clock

- Let event $\mathbf{a}$ be in thread $A$ and event $\mathbf{b}$ be in thread $B$.

- If event $\mathbf{a}$ and event $\mathbf{b}$ are paired synchronization operations, construct a happens-before edge between them:

- E.g. If $a=\operatorname{unlock}(m u)$ and $b=\operatorname{lock}(m u)$ then

$$
\mathrm{a}^{\mathrm{hb}} \rightarrow \mathrm{b} \text { (a happens-before } \mathrm{b} \text { ) }
$$

- Shared accesses $\mathrm{i}$ and $\mathrm{j}$ are concurrent

- if neither $\mathrm{i}$ hb $\rightarrow \mathrm{j}$ nor $\mathrm{j}$ hb $\rightarrow \mathrm{i}$ holds.

- Data races between threads are possible if accesses to shared variables are not ordered by happens-before. 


\section{Happens-Before - Example 1}

- Happens-before analysis will eliminate the false alarm in the following simple case:

\section{$\underline{\text { Thread } 1} \quad \underline{\text { Thread } 2}$ \\ $\mathrm{X}=0$ \\ $\mathrm{X}++$ \\ Signal(CV) \\ Wait(CV) \\ $\mathrm{T}=\mathrm{X}$}




\section{Happens-Before - Example 2}

Thread 1

lock(mu);

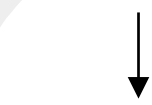

$v=v+1$;

unlock(mu);
Thread 2

$\operatorname{lock}(\mathrm{mu})$;

The arrows represent happens-before. The events represent an actual execution of the two threads. 


\section{Helgrind $^{+}$}

- Efficient hybrid dynamic race detector

- Introduces a new hybrid algorithm based on lockset algorithm and happens-before analysis

- Does runtime analysis and uses code and semantic information

- Different memory state machines for

- short-running applications (during development - unit test)

- More sensitive, but produces more false positives

- long-running applications (integration testing)

- Less sensitive, might miss a race on first iteration, but not on second

- Automatically handling of synchronization bug patterns related to condition variables without any source code annotation

- Lost signal detector

- Spurious wake-up detection 


\section{Ad-hoc (User-defined) Synchronization}

- Synchronization constructs implemented by user for performance reasons

- High level synchronizations (e.g. task queues)

- Spinning read loop instead of a library wait operation

$l^{*}$ Initially FLAG is zero */

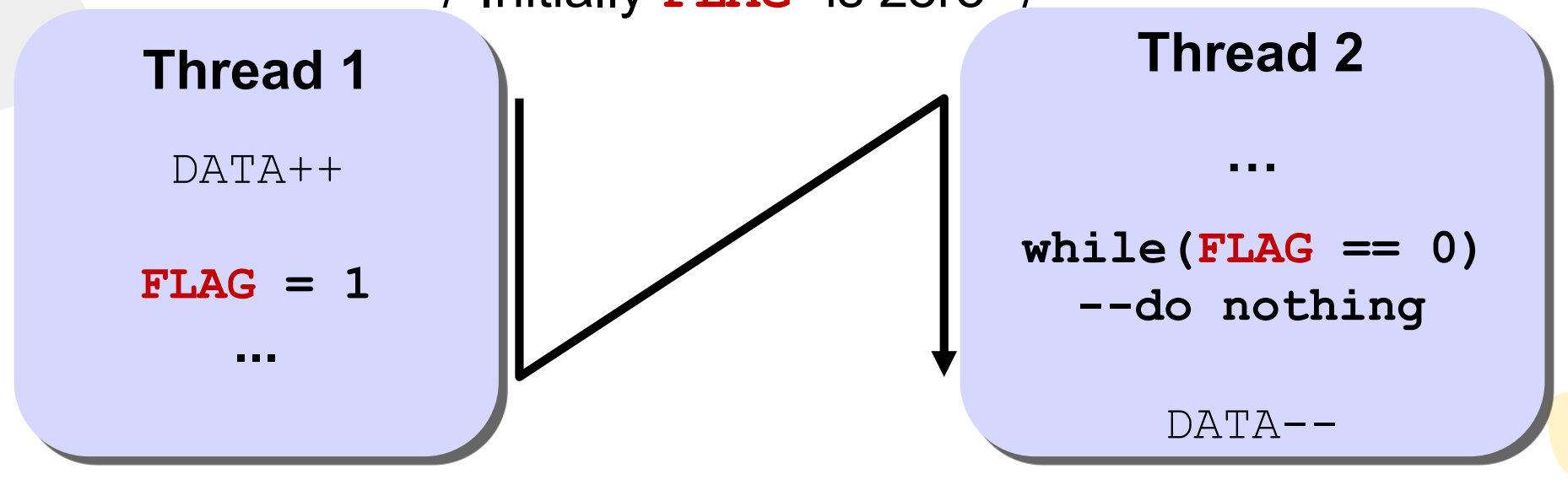

- Ad-hoc synchronizations are widely used

- 12 - 31 in SPLASH-2 and 32 - 329 in PARSEC 2.0 


\section{Ad-hoc Synchronization}

- Source of false positives

- Apparent races (e.g. DATA)

- Synchronization races (e.g. FLAG)

- Detectors should identify and suppress them

- We developed a dynamic method to detect ad-hoc synchronization

- Automatically without any user action

- Capable of identifying synchronization primitives of unknown libraries

- Eliminates false races (apparent and synchronization races) caused by unknown synchronization primitives of a library

- No need to upgrade the detector for a new library 


\section{Common Pattern}

- Spinning read loop (spin-lock) is a common pattern for adhoc synchronizations

- Happens-before relation induced by spin-lock synchronization
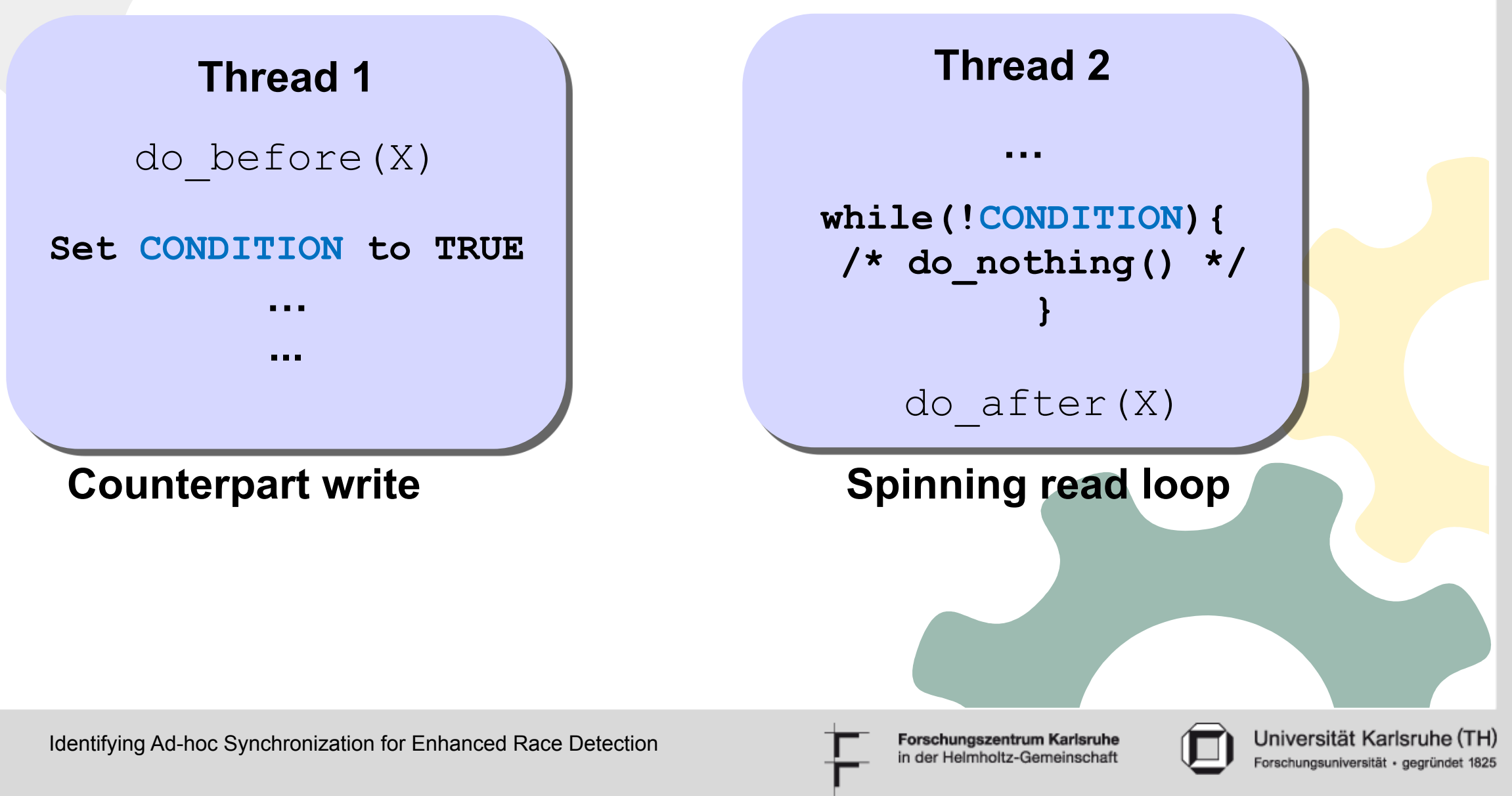


\section{Common Pattern}

- Implementation of different synchronization primitives in libraries follows the same pattern as in spinning read loop

- e.g. implementation of Barrier() :

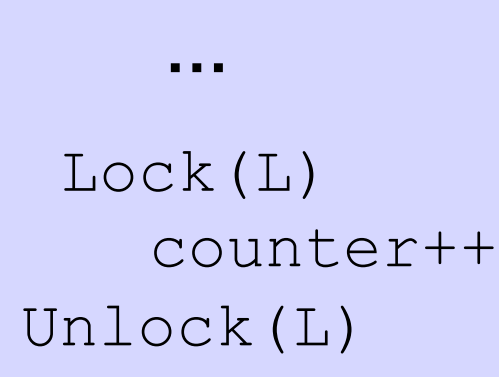

while ( counter ! =NUMBER THREADS) \{ $/ *$ do_nothing () * /

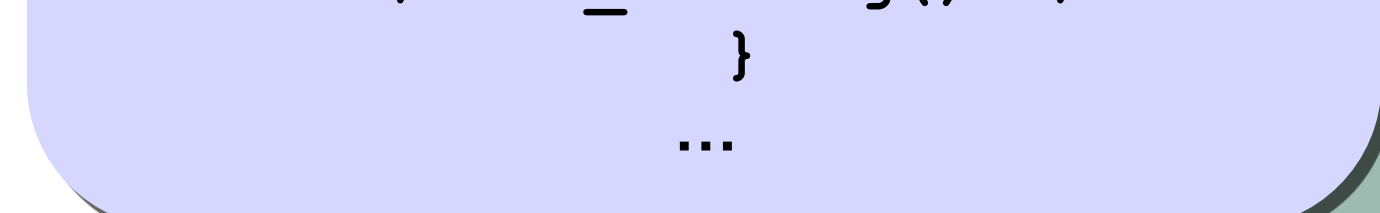




\section{Detecting Ad-hoc Synchronizations}

- General dynamic approach

- Instrumentation phase and

- Runtime phase

- Instrumentation phase (code/semantic analysis)

- Search the binary code to find all loops

- Control flow analysis on the fly

- Consider small loops (3 to 7 basic blocks)

- Detect the spinning read loop based on the following criteria:

- The loop condition involves at least on load instruction from memory

- The value of loop condition is not changed inside the loop

- Instrument the loop and mark the variables that affect the value of the loop condition to be treated specially.

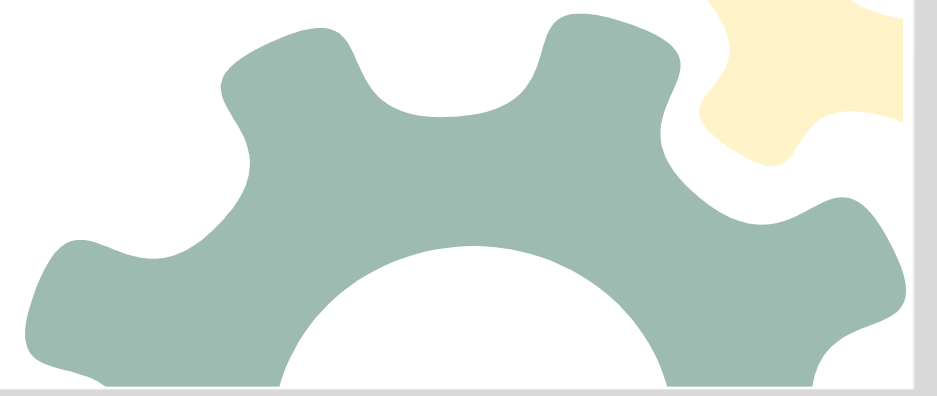




\section{Detecting Ad-hoc Synchronizations}

- Runtime phase

- Data dependency analysis

- Monitor all write/read accesses

- Identify the write/read dependency

- Between the variables of instrumented spinning loop condition and those in counterpart write

- Establish a happens-before relation between corresponding parts

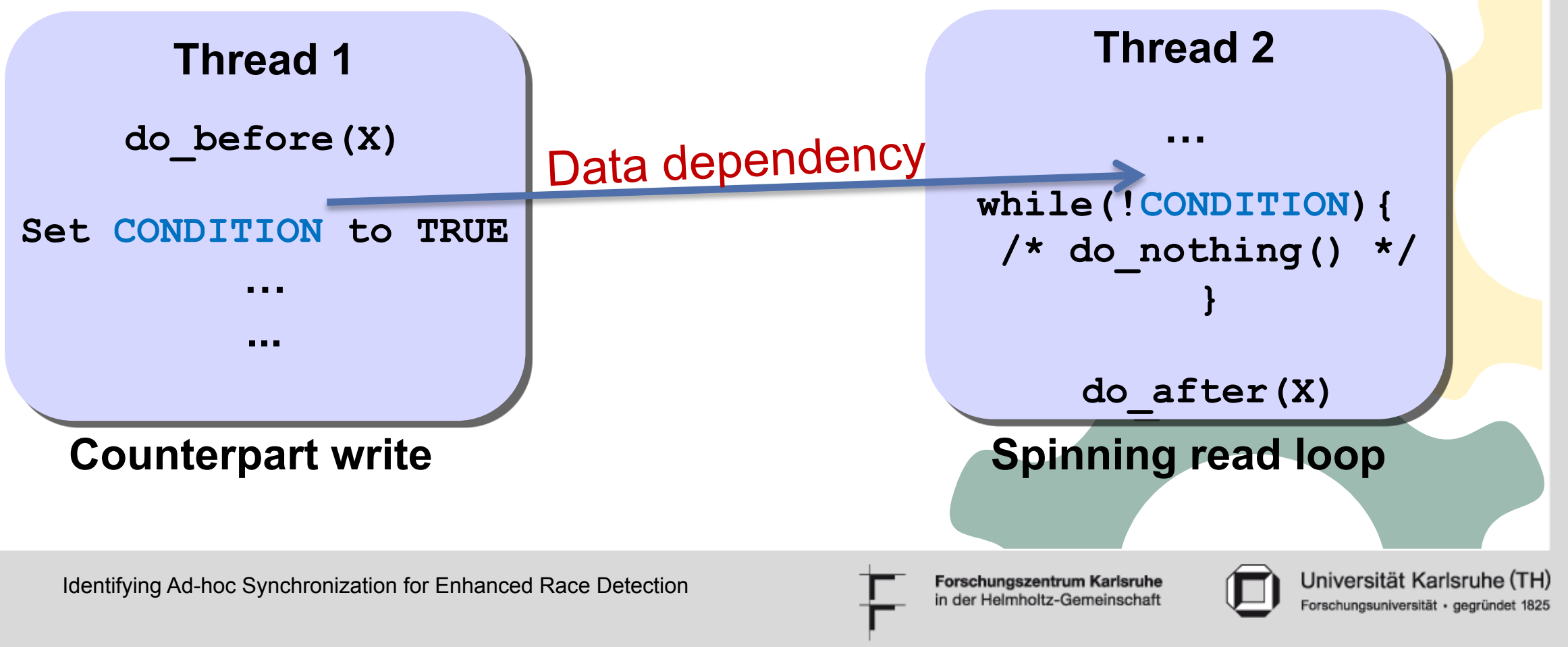




\section{Detecting Unknown Synchronization Primitives}

- Synchronization operations are ultimately implemented by spinning read loops

- Identify unknown synchronization operations if based on spinning read loops.

- If this works, then we actually get a universal race detector

- Not limited to synchronization primitives of a particular library

- General approach to identify synchronization operations

- Information about libraries can be removed entirely from the detector 


\section{Implementation}

- We implement the presented approach into our race detector Helgrind ${ }^{+}$

- Helgrind ${ }^{+}$

- A hybrid dynamic race detector

- Combines lockset algorithm and happens-before analysis

- It is open source and built on top of Valgrind (a binary instrumentation tool) 


\section{Experiments \& Evaluation}

- The approach is evaluated on different benchmarks

- data-race-test - a test suite framework for race detectors

- PARSEC 2.0 Benchmarks

- All experiments were conducted on:

- 2 * $1,86 \mathrm{GHz}$ Xeon E5320 Quadcores, 8 GB RAM

- OS: Linux (Ubuntu 8.10.2)

- New features in Helgrind ${ }^{+}$

- Reduces the number of false positives due to ad-hoc synchronizations and unknown libraries dramatically 


\section{Test Suite - data-race-test}

- 120 different test cases (2-16 Threads)

- Test cases are racy or race-free programs (using Pthread)

- Includes difficult cases

- Spinning read loop detection of up to 7 basic blocks

- 24 false positives and one false negative are removed

- Removing information about Pthread library (unknown library)

- Only one false positive more

\begin{tabular}{|l|c|c|c|c|}
\hline Tools & $\begin{array}{l}\text { False } \\
\text { alarms }\end{array}$ & $\begin{array}{l}\text { Missed } \\
\text { races }\end{array}$ & $\begin{array}{l}\text { Failed } \\
\text { cases }\end{array}$ & $\begin{array}{l}\text { Correctly } \\
\text { analyzed } \\
\text { cases }\end{array}$ \\
\hline Helgrind ${ }^{+}$lib & 32 & 8 & 40 & 80 \\
\hline Helgrind $^{+}$lib+spin(7) & $\mathbf{8}$ & $\mathbf{7}$ & $\mathbf{1 5}$ & $\mathbf{1 0 5}$ \\
\hline Helgrind ${ }^{+}$nolib+spin(7) & 9 & 7 & 16 & 104 \\
\hline DRD & 13 & 20 & 33 & 87 \\
\hline \multicolumn{5}{|l|}{} \\
Identifing Ad-hoc Synchrorization for Enhanced Race Detection
\end{tabular}


Test Suite - data-race-test

- Best result achieved with seven basic blocks using spinning read loop detection as a complementary method

- In most cases spinning read loops contain more than 3 basic blocks

- loop conditions use templates and complex function calls

\begin{tabular}{|l|c|c|c|c|}
\hline Tools & $\begin{array}{l}\text { False } \\
\text { alarms }\end{array}$ & $\begin{array}{l}\text { Missed } \\
\text { races }\end{array}$ & $\begin{array}{l}\text { Failed } \\
\text { cases }\end{array}$ & $\begin{array}{l}\text { Correctly } \\
\text { analysed } \\
\text { cases }\end{array}$ \\
\hline Helgrind $^{+}$lib+spin(3) & 24 & 7 & 31 & 89 \\
\hline Helgrind $^{+}$lib+spin(6) & 23 & 7 & 30 & 90 \\
\hline Helgrind $^{+}$lib+spin(7) & $\mathbf{8}$ & $\mathbf{7}$ & $\mathbf{1 5}$ & $\mathbf{1 0 5}$ \\
\hline Helgrind $^{+}$lib+spin(8) & 8 & 7 & 15 & 105 \\
\hline
\end{tabular}


PARSEC 2.0

\begin{tabular}{|c|c|c|c|c|c|c|}
\hline \multirow{2}{*}{ Program } & \multirow{2}{*}{$\begin{array}{c}\text { Parallelization } \\
\text { model }\end{array}$} & \multirow{2}{*}{ LOC } & \multicolumn{3}{|c|}{ Synchronisation primitives } & \multirow{2}{*}{ Ad-hoc } \\
\hline & & & CVs & Locks & Barriers & \\
\hline blackscholes & POSIX & 812 & - & - & $\checkmark$ & - \\
\hline swaptions & POSIX & 4,029 & - & - & - & - \\
\hline fluidanimate & POSIX & 3,689 & - & $\checkmark$ & - & - \\
\hline canneal & POSIX & 29,31 & - & $\checkmark$ & - & - \\
\hline freqmine & OpenMP & 10,279 & - & - & - & - \\
\hline vips & GLIB & 1,255 & $\checkmark$ & $\checkmark$ & - & $\checkmark$ \\
\hline bodytrack & POSIX & 9,735 & $\checkmark$ & $\checkmark$ & $\checkmark$ & $\checkmark$ \\
\hline facesim & POSIX & 1,391 & $\checkmark$ & $\checkmark$ & - & $\checkmark$ \\
\hline ferret & POSIX & 2,706 & $\checkmark$ & $\checkmark$ & - & $\checkmark$ \\
\hline$x 264$ & POSIX & 1,494 & $\checkmark$ & $\checkmark$ & - & $\checkmark$ \\
\hline dedup & POSIX & 3,228 & $\checkmark$ & $\checkmark$ & - & $\checkmark$ \\
\hline streamcluster & POSIX & 40,393 & $\checkmark$ & $\checkmark$ & $\checkmark$ & $\checkmark$ \\
\hline raytrace & POSIX & 13,302 & $\checkmark$ & $\checkmark$ & - & $\checkmark$ \\
\hline
\end{tabular}




\section{Programs without Ad-hoc Synchronizations}

- No false positives for first 4 programs

- In case of using the unknown library OpenMP only 2 false positives remain

\begin{tabular}{|l|l|c|c|c|c|c|}
\hline \multirow{2}{*}{ Program } & \multirow{2}{*}{$\begin{array}{c}\text { Para. } \\
\text { model }\end{array}$} & \multirow{2}{*}{ LOC } & \multicolumn{5}{|c|}{ Racy Contexts } \\
\cline { 4 - 8 } & & $\begin{array}{c}\text { Helgrind } \\
\text { lib }\end{array}$ & $\begin{array}{c}\text { Helgrind } \\
\text { lib+spin }\end{array}$ & $\begin{array}{c}\text { Helgrind+ } \\
\text { nolib+spin }\end{array}$ & DRD \\
\hline blackscholes & POSIX & 812 & 0 & $\mathbf{0}$ & 0 & 0 \\
\hline swaptions & POSIX & 4,029 & 0 & $\mathbf{0}$ & 0 & 0 \\
\hline fluidanimate & POSIX & 3,689 & 0 & $\mathbf{0}$ & 0 & 0 \\
\hline canneal & POSIX & 29,31 & 0 & $\mathbf{0}$ & 0 & 0 \\
\hline freqmine & OpenMP & 10,279 & 153.4 & $\mathbf{2}$ & 2 & 1000 \\
\hline
\end{tabular}




\section{Programs with Ad-hoc Synchronizations}

- In 5 out of 8 programs false positives are completely eliminated

\begin{tabular}{|l|l|l|l|c|c|c|}
\hline \multirow{2}{*}{ Program } & \multirow{2}{*}{$\begin{array}{c}\text { Para. } \\
\text { model }\end{array}$} & LOC & \multicolumn{4}{|c|}{ Racy Contexts } \\
\cline { 5 - 8 } & & $\begin{array}{c}\text { Helgrind } \\
\text { lib }\end{array}$ & $\begin{array}{c}\text { Helgrind } \\
\text { lib+spin }\end{array}$ & $\begin{array}{c}\text { Helgrind } \\
\text { nolib+spin }\end{array}$ & DRD \\
\hline vips & GLIB & 1,255 & 50.8 & $\mathbf{0}$ & 0 & 858.6 \\
\hline bodytrack & POSIX & 9,735 & 36.8 & $\mathbf{3 . 6}$ & 32.4 & 34.6 \\
\hline facesim & POSIX & 1,391 & 113.8 & $\mathbf{0}$ & 0 & 1000 \\
\hline ferret & POSIX & 2,706 & 111 & $\mathbf{2}$ & 47 & 214.6 \\
\hline x264 & POSIX & 1,494 & 1000 & $\mathbf{1 9}$ & 28 & 1000 \\
\hline dedup & POSIX & 3,228 & 1000 & $\mathbf{0}$ & 2 & 0 \\
\hline streamcluster & POSIX & 40,393 & 4 & $\mathbf{0}$ & 1 & 1000 \\
\hline raytrace & POSIX & 13,302 & 106,4 & $\mathbf{0}$ & 0 & 1000 \\
\hline
\end{tabular}




\section{Programs with Ad-hoc Synchronizations}

- 3 programs produce false positives (2 to 19 warnings)

- Function pointers for condition evaluation and obscure implementation of task queue (do not match the spin patterns)

\begin{tabular}{|l|l|l|l|c|c|c|}
\hline \multirow{2}{*}{ Program } & \multirow{2}{*}{$\begin{array}{c}\text { Para. } \\
\text { model }\end{array}$} & LOC & \multicolumn{5}{|c|}{ Racy Contexts } \\
\cline { 5 - 7 } & & $\begin{array}{c}\text { Helgrind } \\
\text { lib }\end{array}$ & $\begin{array}{c}\text { Helgrind+ }^{+} \\
\text {lib+spin }\end{array}$ & $\begin{array}{c}\text { Helgrind }^{+} \\
\text {nolib+spin }\end{array}$ & DRD \\
\hline vips & GLIB & 1,255 & 50.8 & $\mathbf{0}$ & 0 & 858.6 \\
\hline bodytrack & POSIX & 9,735 & 36.8 & $\mathbf{3 . 6}$ & 32.4 & 34.6 \\
\hline facesim & POSIX & 1,391 & 113.8 & $\mathbf{0}$ & 0 & 1000 \\
\hline ferret & POSIX & 2,706 & 111 & $\mathbf{2}$ & 47 & 214.6 \\
\hline x264 & POSIX & 1,494 & 1000 & $\mathbf{1 9}$ & 28 & 1000 \\
\hline dedup & POSIX & 3,228 & 1000 & $\mathbf{0}$ & 2 & 0 \\
\hline streamcluster & POSIX & 40,393 & 4 & $\mathbf{0}$ & 1 & 1000 \\
\hline raytrace & POSIX & 13,302 & 106,4 & $\mathbf{0}$ & 0 & 1000 \\
\hline
\end{tabular}


Universal Race Detector

\begin{tabular}{|c|c|c|c|c|c|c|}
\hline \multirow{2}{*}{ Program } & \multirow{2}{*}{$\begin{array}{l}\text { Para. } \\
\text { model }\end{array}$} & \multirow[b]{2}{*}{ LOC } & \multicolumn{4}{|c|}{ Racy Contexts } \\
\hline & & & $\begin{array}{l}\text { Helgrind } \\
\text { lib }\end{array}$ & $\begin{array}{l}\text { Helgrind }{ }^{+} \\
\text {lib+spin }\end{array}$ & $\begin{array}{c}\text { Helgrind }^{+} \\
\text {nolib+spin }\end{array}$ & DRD \\
\hline \multicolumn{5}{|c|}{ Happens-before detector $n$ O } & 0 & 0 \\
\hline \multirow{2}{*}{\multicolumn{3}{|c|}{$\begin{array}{l}\text { false positives are } \\
\text { slightly increased } \\
\text { in } 4 \text { cases }\end{array}$}} & 0 & 0 & 0 & 0 \\
\hline & & & 0 & 0 & 0 & 0 \\
\hline canneal & POSIX & 29,31 & 0 & 0 & 0 & 0 \\
\hline freqmine & OpenMP & 10,279 & 153.4 & 2 & 2 & 1000 \\
\hline vips & GLIB & 1,255 & 50.8 & 0 & 0 & 858.6 \\
\hline bodytrack & POSIX & 9,735 & 36.8 & 3.6 & 32.4 & 34.6 \\
\hline facesim & POSIX & 1,391 & 113.8 & 0 & 0 & 1000 \\
\hline ferret & POSIX & 2,706 & 111 & 2 & 47 & 214.6 \\
\hline$x 264$ & POSIX & 1,494 & 1000 & 19 & 28 & 1000 \\
\hline dedup & POSIX & 3,228 & 1000 & 0 & 2 & 0 \\
\hline streamcluster & POSIX & 40,393 & 4 & 0 & 1 & 1000 \\
\hline raytrace & POSIX & 13,302 & 106,4 & 0 & 0 & 1000 \\
\hline
\end{tabular}




\section{Performance}

- Minor overhead due to the new feature for spinning read detection

- Memory consumption:

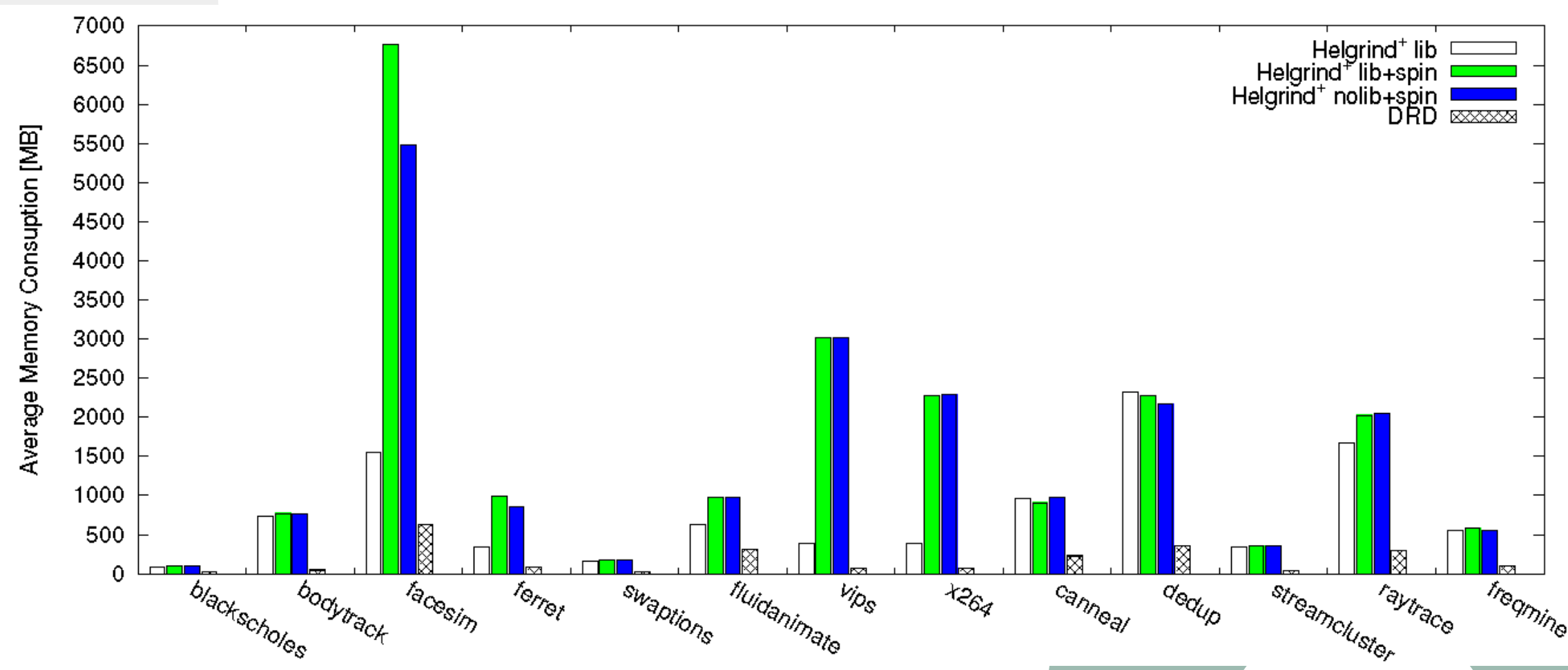




\section{Performance}

- Slight runtime overhead:

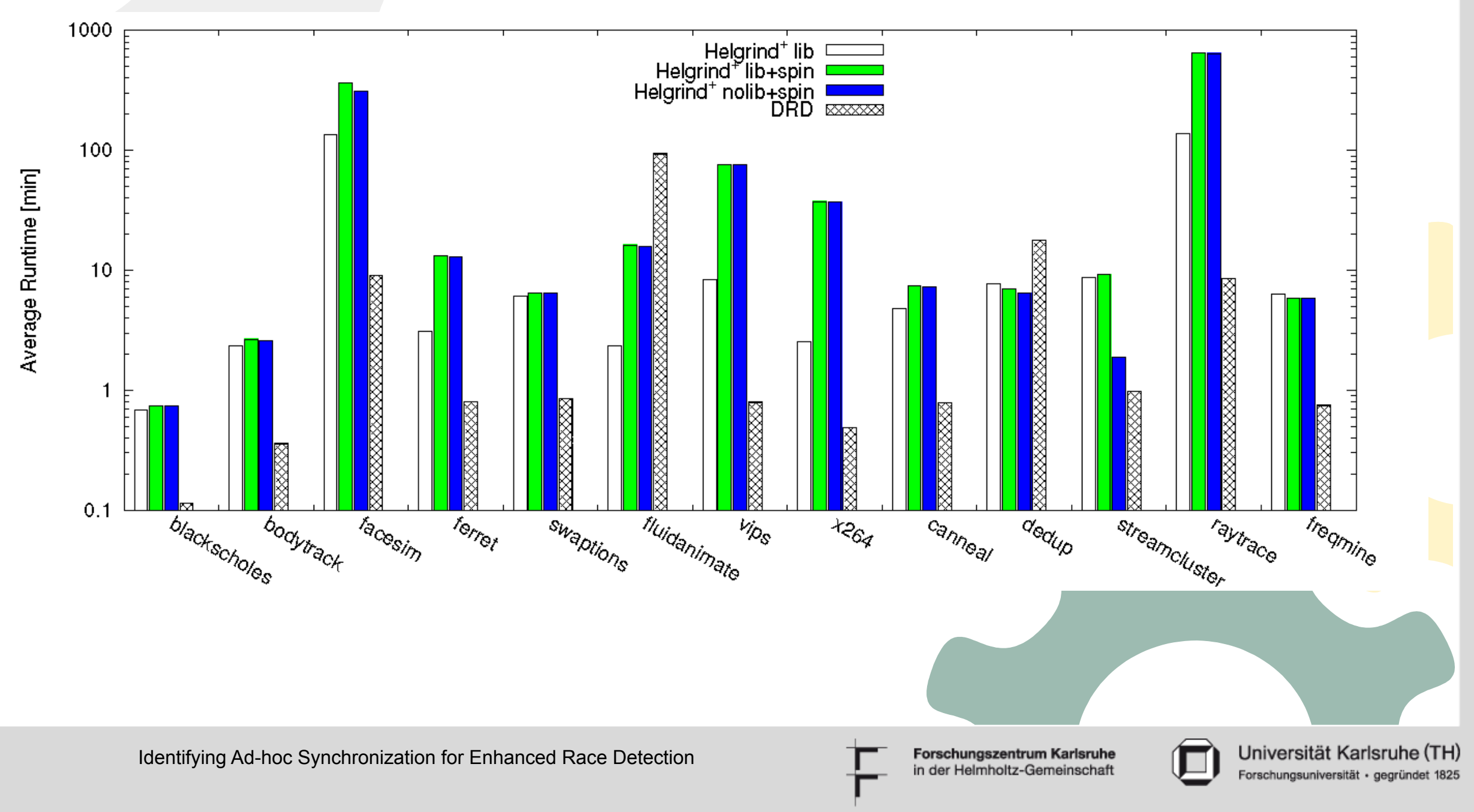




\section{Summary}

- Knowledge of all synchronization operations are crucial for accurate data race detection

- Missing ad-hoc synchronizations causes a lot of false positives

- We present a dynamic method that is able to identify adhoc and unknown synchronizations in programs

- Universal race Detector

- No need to upgrade the detector for unknown libraries

- Best results achieved when using it as complementary method (applicable for every race detector)

- Future work: Improving the accuracy of the universal race detector by identifying the lock operations (enabling lockset analysis). 


\section{Thank you}

\section{Questions?}

This work: Ali Jannesari, Walter F. Tichy, Identifying Ad-hoc Synchronization for Enhanced Race Detection, to appear in International Parallel \& Distributed Processing Symposium (IPDPS'10), Apr 2010.

Helgrind+: Ali Jannesari, Kaibin Bao, Victor Pankratius, Walter F. Tichy, Helgrind+: An Efficient Dynamic Race Detector, Proceedings of the 23rd international Parallel \& Distributed Processing Symposium (IPDPS'09), 2009.

www.ipd.uka.de/Tichy/ 